\title{
On the Supersymmetry of Non-BPS D-brane
}

\author{
Seiji Terashima円 and Tadaoki Uesugi[ \\ Department of Physics, Faculty of Science \\ University of Tokyo \\ Tokyo 113-0033, Japan
}

\begin{abstract}
In this paper we extend the boundary string field theory action for a non-BPS D-brane to the one including the target space fermions and the nonlinear supersymmetry with 32 supercharges up to some order. This is based on the idea that the vacuum with a non-BPS D-brane belongs to the spontaneously broken phase of the supersymmetry. As a result, we find that the action is almost uniquely determined up to the field redefinition ambiguities.
\end{abstract}

\footnotetext{
${ }^{1}$ seiji@hep-th.phys.s.u-tokyo.ac.jp

${ }^{2}$ uesugi@hep-th.phys.s.u-tokyo.ac.jp
} 


\section{Introduction}

The supersymmetry is one of the most important subjects to know the full structure of string theory. In fact the tremendous progress of understanding the non-perturbative aspects of string theory has relied on the supersymmetry. In RNS formalism such a ten dimensional supersymmetry in various supersymmetric string theories is induced by the superconformal invariance and GSO-projection on the string sigma model action. However, we do not understand why the supersymmetry naturally appears in the string theory and why such prescriptions make it possible to introduce the supersymmetry. What is the connection between two dimensional superconformal symmetry and ten dimensional supersymmetry? What is the mathematical and the physical meanings of GSO-projections?

On the other hand, recently there has been a lot of studies (for example, see [1, 2]) on the non-BPS physics with different motivations from the above arguments, and the off-shell dynamics of non-BPS systems has been possible to discuss. The off-shell actions on non-BPS systems are the most important quantities which can be obtained from the various string field theories. Especially by using boundary string field theory [3, 4, 5], the exact actions for non-BPS D-branes and brane-antibrane systems has been recently computed [6, 7, 8, 9]. Unfortunately the string field theories including the R-sector have not been found until now, therefore these actions are written only by the bosonic fields.

From these actions we can understand various dynamical aspects of non-BPS systems. For example, a non-BPS D-brane decays to the closed string vacuum by the trivial tachyon condensation or to a BPS D8-brane by the one with the topological defect. This fact means that the vacuum with the supersymmetry is connected to the vacuum with the non-BPS D-brane. Then it is natural to consider that the vacuum with non-BPS D-branes belongs to the spontaneously broken phase of the supersymmetry in type II theories [10, 11]. If so, in the field theory on a non-BPS D-brane the nonlinear supersymmetry should be realized. This is a interesting subject because in non-gravitational field theory with 32 supercharges the linear supersymmetry is not known. Moreover there is no GSO-projection on the fields which are excited by open strings, thus from this we may understand the relation between GSO-projection and the supersymmetry.

The supersymmetry with 32 supercharges is very large, thus we expect that only this symmetry gives enough constraints to determine the action almost completely. Then, as a first step to understand this, we try to extend the field theory action of the boundary string field theory on a non-BPS D-brane to the one with target space fermions which has the non-linear supersymmetry.

In the next section we review the present understandings of the supersymmetry on a non-BPS D-brane, and in section 3 we explicitly construct the field theory action with nonlinear supersymmetry by considering all possible terms in the action, supersymmetry transformations and field redefinitions up to some order. This action is unique up to the 
field redefinition ambiguities. The conclusion and the discussion are in section 4 .

\section{Supersymmetry on a Non-BPS D-brane}

First we review the various arguments about the supersymmetry on a non-BPS D-brane. In this paper we consider the non-BPS D9-brane in type IIA superstring theory. If we need to consider the non-BPS D $p$-brane, we have only to perform the T-duality, therefore it is enough to consider the D9-brane.

On a non-BPS D9-brane there are infinite numbers of the fields which are excited by the open strings without GSO-projection. They are massive fields, a U(1) gauge field, a non-chiral Majorana fermion of $\mathrm{SO}(1,9)$ and a real tachyon. The tachyon and the Majorana fermion belong to the adjoint representation for the $\mathrm{U}(1)$ gauge transformation, that is, they are gauge-singlets. Formally we can define a effective action written by all these fields on the non-BPS D-brane, however in general for our convenience we integrate out the massive fields which we do not need to consider[.

In 10] the author gives the action with the massless fermion in which the tachyon is integrated out. Its action is 1

$$
\begin{aligned}
S & =-\sqrt{2} T_{9} \int d^{10} x \sqrt{-\operatorname{det}\left(\eta_{\mu \nu}+\mathcal{F}_{\mu \nu}\right)}, \\
& =-\sqrt{2} T_{9} \int d^{10} x\left[1+\frac{1}{4} F^{\mu \nu} F_{\mu \nu}-\bar{\psi} \Gamma^{\mu} \partial_{\mu} \psi+F^{\mu \nu}\left(\bar{\psi} \Gamma_{11} \Gamma_{\mu} \partial_{\nu} \psi\right)+\cdots\right],
\end{aligned}
$$

where

$$
\begin{aligned}
\mathcal{F}_{\mu \nu}= & F_{\mu \nu}-\left(\bar{\psi} \Gamma_{\nu} \partial_{\mu} \psi\right)-\left(\bar{\psi} \Gamma_{\mu} \partial_{\nu} \psi\right) \\
& -\left(\bar{\psi} \Gamma_{11} \Gamma_{\nu} \partial_{\mu} \psi\right)+\left(\bar{\psi} \Gamma_{11} \Gamma_{\mu} \partial_{\nu} \psi\right)+\left(\bar{\psi} \Gamma^{\rho} \partial_{\mu} \psi\right)\left(\bar{\psi} \Gamma_{\rho} \partial_{\nu} \psi\right) \\
& +\frac{1}{2}\left(\bar{\psi} \Gamma_{11} \Gamma_{\rho} \partial_{\mu} \psi\right)\left(\bar{\psi} \Gamma^{\rho} \partial_{\nu} \psi\right)-\frac{1}{2}\left(\bar{\psi} \Gamma_{11} \Gamma_{\rho} \partial_{\nu} \psi\right)\left(\bar{\psi} \Gamma^{\rho} \partial_{\mu} \psi\right) .
\end{aligned}
$$

Here we write the effective action whose diffeomorphism invariance is already fixed by the static gauge [10].

However, the most characteristic property of this action is that this action has the nonlinear supersymmetry with 32 supercharges. The transformations are as follows:

$$
\begin{aligned}
\delta \psi_{\alpha}= & \epsilon_{\alpha}-\left(\bar{\epsilon} \Gamma^{\mu} \psi\right)\left(\partial_{\mu} \psi\right)_{\alpha} \\
\delta A_{\mu}= & \left(\bar{\epsilon} \Gamma_{11} \Gamma_{\mu} \psi\right)-\left(\bar{\epsilon} \Gamma^{\nu} \psi\right) \partial_{\nu} A_{\mu}-\left(\bar{\epsilon} \Gamma^{\rho} \partial_{\mu} \psi\right) A_{\rho} \\
& -\frac{1}{6}\left\{\left(\bar{\epsilon} \Gamma_{11} \Gamma_{\nu} \psi\right)\left(\bar{\psi} \Gamma^{\nu} \partial_{\mu} \psi\right)+\left(\bar{\epsilon} \Gamma_{\nu} \psi\right)\left(\bar{\psi} \Gamma_{11} \Gamma^{\nu} \partial_{\mu} \psi\right)\right\} .
\end{aligned}
$$

\footnotetext{
3"Integrate out" means by inserting the solution to the equation of motion of the field because here we consider the effective action only at the tree level.

${ }^{4}$ In this paper we set $2 \pi \alpha^{\prime}$ to 1 for simplicity.
} 
From these transformations we can obtain the following supersymmetry algebra:

$$
\left[\delta_{1}, \delta_{2}\right] \psi_{\alpha}=2\left(\bar{\epsilon}_{1} \Gamma^{\rho} \epsilon_{2}\right) \partial_{\rho} \psi_{\alpha} \quad, \quad\left[\delta_{1}, \delta_{2}\right] A_{\mu}=-2\left(\bar{\epsilon}_{1} \Gamma_{11} \Gamma_{\mu} \epsilon_{2}\right)+2\left(\bar{\epsilon}_{1} \Gamma^{\rho} \epsilon_{2}\right) \partial_{\rho} A_{\mu}
$$

Thus, we can obtain the expected translations together with a constant shift of the gauge field which can be regarded as an irrelevant gauge transformation. This nonlinear supersymmetry realizes the idea that the vacuum with a non-BPS D-brane belongs to the spontaneously broken phase of the 32 supersymmetries of type II theories. The evidence of the action (2.1) and the supersymmetry transformation (2.3) is given in [12 with the explicit calculations of the on-shell scattering amplitudes.

The configurations where this supersymmetry restores are the closed string vacuum or the vacuum with BPS D-branes. At these configurations the total or partial supersymmetries are linearly represented. These stable vacuum and unstable one with the non-BPS D-brane are connected by the process of the tachyon condensation. Therefore if we want to consider the off-shell dynamics of non-BPS D-branes and realize this supersymmetry at the arbitrary off-shell point, we have to incorporate the tachyon field. Namely, we need the effective action before the tachyon is integrated out. Therefore from (2.1) we can not see the mechanism of the restoration of the supersymmetry.

On the other hand, recently the effective action which is represented only by the bosonic fields including the tachyon $(\psi(x)$ is set to zero) has been obtained by the open string field theory. Especially, some exact results on the effective action of the various non-BPS systems have been derived from the boundary string field theory [6, 7, 8, 9]. The action for a non-BPS D-brane [13, 6] is

$$
\begin{aligned}
S & =-\sqrt{2} T_{9} \int d^{10} x e^{-\frac{1}{4} T^{2}} \frac{\prod_{r=\frac{1}{2}}^{\infty} \operatorname{det}\left(\eta_{\mu \nu}+F_{\mu \nu}+\frac{1}{4 \pi r} \partial_{\mu} T \partial_{\nu} T\right)}{\prod_{m=1}^{\infty} \operatorname{det}\left(m \eta_{\mu \nu}+m F_{\mu \nu}+\frac{1}{4 \pi} \partial_{\mu} T \partial_{\nu} T\right)} \\
& =-\sqrt{2} T_{9} \int d^{10} x e^{-\frac{1}{4} T^{2}} \sqrt{-\operatorname{det}\left(\eta_{\mu \nu}+F_{\mu \nu}\right)} \mathcal{F}\left[\frac{1}{4 \pi} \mathcal{G}^{\mu \nu} \partial_{\mu} T \partial_{\nu} T\right]
\end{aligned}
$$

where

$$
\mathcal{G}^{\mu \nu} \equiv\left(\frac{1}{1+F}\right)^{(\mu \nu)} \quad, \quad \mathcal{F}[x] \equiv \frac{4^{x} x(\Gamma(x))^{2}}{2 \Gamma(2 x)}=1+2(\log 2) x+O\left(x^{2}\right) .
$$

Here, $(\mu \nu)$ indicates the symmetrization. The "exactness" of this action means that it is

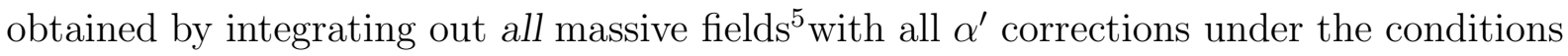
that $\partial_{\mu} \partial_{\nu} T=0$, and $\partial_{\rho} F_{\mu \nu}=0$. 宁

\footnotetext{
${ }^{5}$ In the other string field theory it is difficult to perform this, therefore the approximation is used which is usually called level truncation.

${ }^{6}$ However, even if we regard (2.5) as the complete action without this condition, the action seems to give several nontrivial results. For example, in 14] the author constructed the various soliton solutions which do not obey the above conditions.
} 
As an example of the tachyon condensation in (2.5) we consider the following nontrivial exact soliton solutions which represents a BPS D8-brane localized at $x^{9}=0$ after the tachyon condensation [6]:

$$
T(x)=u x^{9} \quad(u \rightarrow \infty) \quad, \quad A_{\mu}(x)=0 \quad, \quad \psi(x)=0
$$

This solution gives a correct tension and a D8-brane charge from the action (2.5) and Wess-Zumino term of the non-BPS D-brane [8, 9]. These facts give the evidences for the identification of this solution as a BPS D8-brane.

From these two actions (2.1) and (2.5) we expect that in boundary string field theory action (2.5) the nonlinear supersymmetry can be realized by including the transformations not only of the gauge field and the fermion but also of the tachyon. Indeed we can prove the existence of such an action and supersymmetry transformations. First we define $S_{0}\left(C^{a}, \lambda^{i}\right)$ as an action where not only $T(x), \psi(x)$ and $A_{\mu}(x)$ (here, we represent these three fields as $\left.C^{a}(x)(a=1,2,3)\right)$ but also the massive fields $\lambda^{i}(x)$ are included. The supersymmetric invariance of the action is written by

$$
S_{0}\left(C^{a}+\delta C^{a}, \lambda^{i}+\delta \lambda^{i}\right)=S_{0}\left(C^{a}, \lambda^{i}\right) \quad \text { where } \quad\left\{\begin{aligned}
\delta C^{a} & =\delta C^{a}\left(C^{a}, \lambda^{i}\right) \\
\delta \lambda^{i} & =\delta \lambda^{i}\left(C^{a}, \lambda^{i}\right) .
\end{aligned}\right.
$$

Then, we integrate out the massive fields $\lambda^{i}(x)$ from $S_{0}\left(C^{a}, \lambda^{i}\right)$. This is performed by inserting the solution to the equation of motion for $\lambda^{i}(x)$. If we define $\bar{\lambda}^{i}=\bar{\lambda}^{i}\left(C^{a}\right)$ as the solution to the equation of motion for $\lambda^{i}(x)$ and insert this solution into (2.8), then the result is

$$
\begin{aligned}
S_{0}\left(C^{a}, \bar{\lambda}^{i}\right) & =S_{0}\left(C^{a}+\delta C^{a}\left(C^{a}, \bar{\lambda}^{i}\right), \bar{\lambda}^{i}+\delta \lambda^{i}\left(C^{a}, \bar{\lambda}^{i}\right)\right) \\
& =S_{0}\left(C^{a}+\delta C^{a}\left(C^{a}, \bar{\lambda}^{i}\right), \bar{\lambda}^{i}\right) .
\end{aligned}
$$

Here we used the relation $\left.\frac{\delta S_{0}}{\delta \lambda^{i}}\right|_{\lambda=\bar{\lambda}}=0$. If we define the action in which the massive fields are integrated out as $S\left(C^{a}\right) \equiv S_{0}\left(C^{a}, \bar{\lambda}^{i}\left(C^{a}\right)\right)$, the above relation indicates that $S\left(C^{a}\right)$ is invariant for $C^{a} \rightarrow C^{a}+\bar{\delta} C^{a}$ where $\bar{\delta} C^{a}\left(C^{a}\right) \equiv \delta C^{a}\left(C^{a}, \bar{\lambda}^{i}\left(C^{a}\right)\right)$.

This conjecture of the realization of the nonlinear supersymmetry with 32 supercharges is highly nontrivial because, regardless of the dimension, in the supersymmetric field theory with 32 supercharges any supermultiplets can not be constructed without introducing the graviton. This indicates that we can not construct any field theories with the linear supersymmetry. However, the nonlinear realization of the supersymmetry with 32 supercharges is an exception we can consider to avoid this no-go theorem.

In (2.3) the supersymmetry transformations have been already obtained, however, from these equations we can not discuss the tachyon condensation. If we can obtain the action without integrating out the tachyon field, we can understand the mechanism of the restoration of the linear supersymmetry through the analysis of the tachyon condensation. 
The idea of the realization of the nonlinear supersymmetry in the off-shell region was considered in [1] by using Witten's cubic type open superstring field theory [17]. In this paper the author generalized this theory to the string field theory on the non-BPS D-brane by including the GSO-odd components of the string field, and constructed the nonlinear supersymmetry transformations of the string field. However, Witten's superstring field theory has a problem about the collision of picture changing operators, thus this discussion is rather formal. Moreover in this theory the explicit BPS solution has been not obtained until now, therefore we can not see how the linear supersymmetry restores at this stable vacuum.

On the other hand, contrary to this theory, the recent work on the boundary string field theory has given the exact action (2.5) and the exact soliton solution (2.7). Therefore by using these results we expect that the nonlinear supersymmetry can be realized explicitly in the boundary string field theory action (2.5) which will become the action (2.1) with integrating out the tachyon. If so, we can see the restoration of the linear supersymmetry after the tachyon condensation. Concretely speaking, the solution (2.7) is expected to be a BPS solution in the sense of the supersymmetric field theory, that is, this solution will satisfy the BPS equation $\delta_{S U S Y} \psi(x)=0$. If we expand the action around this solution and integrate out the massive fields, the result is expected to become the supersymmetric Dirac-Born-Infeld action for a BPS D-brane [15, 16].

Of course, without the knowledge of the string field theory with R-sector, it may be difficult to obtain the full action which includes all terms with fermions. However, in the supersymmetric quantum mechanics (for example, see [18) there is an interesting example of the model:

$$
S=\int d t e^{-\frac{T^{2}}{4}}\left[\frac{1}{2}\left(\partial_{t} T\right)^{2}-1+\frac{i}{2} \psi^{+} \stackrel{\leftrightarrow}{\partial}_{t} \psi^{-}+\frac{T}{2 \sqrt{2}} \psi^{+} \psi^{-}\right] .
$$

And this action is invariant by the following nonlinear supersymmetry transformations:

$$
\begin{aligned}
\delta T & =i\left(\psi^{+} \eta^{-}+\psi^{-} \eta^{+}\right) \\
\delta \psi^{+} & =-i \sqrt{2} \eta^{+}-\partial_{t} T \eta^{+}+\frac{i}{4} T \psi^{+} \psi^{-} \eta_{,}^{+} \delta \psi^{-}=i \sqrt{2} \eta^{-}-\partial_{t} T \eta^{-}-\frac{i}{4} T \psi^{+} \psi^{-} \eta^{-}
\end{aligned}
$$

and these transformations satisfy the usual supersymmetry algebra. This is equivalent to a well known action in supersymmetric quantum mechanics:

$$
\begin{gathered}
S=2 \pi \int d t\left[\frac{1}{2} \dot{\phi}^{2}-\frac{1}{2} W^{2}(\phi)-W^{\prime}(\phi) \theta^{+} \theta^{-}+i \theta^{+} \dot{\theta}^{-}\right] . \\
\delta \phi=i\left(\theta^{+} \eta^{-}+\theta^{-} \eta^{+}\right), \quad \delta \theta^{ \pm}=\mp i(W(\phi) \mp i \dot{\phi}) \eta^{ \pm},
\end{gathered}
$$

where $\phi=\frac{2}{\sqrt{\pi}} \int_{0}^{\frac{T}{2 \sqrt{2}}} e^{-s^{2}} d s, W(\phi)=\frac{1}{\sqrt{\pi}} \exp \left(-\frac{1}{8} T(\phi)^{2}\right)$ and $\theta^{ \pm}=(2 \pi)^{-\frac{1}{2}} e^{-\frac{T^{2}}{8}} \psi^{ \pm}$. This action shows that the nonlinear supersymmetry can be included for the action with the 
boundary string field theory potential $\exp \left(-\frac{T^{2}}{4}\right)$ at least in one dimension. At $T= \pm \infty$ the superpotential vanishes, that is $W=0$, and the supersymmetry restores as we can see from (2.13). Moreover this has BPS solutions $T= \pm \sqrt{2} t_{E}$ if we perform the Wick rotation $t \rightarrow-i t_{E}$ 凹.

Of course the extension of this model to the one in ten dimension is nontrivial because the field theory on a non-BPS D-brane has infinite number of terms and because in (2.10) the gauge field on a non-BPS D9-brane is not included. However, there is a truncated model which approximately describes the tachyon condensation on a non-BPS D-brane [19]. The action is

$$
S=-\sqrt{2} T_{9} \int d^{10} x e^{-\frac{1}{4} T^{2}}\left[\frac{1}{2} \partial^{\mu} T \partial_{\mu} T+1+\frac{1}{4} F^{\mu \nu} F_{\mu \nu}-\bar{\psi} \Gamma^{\mu} \partial_{\mu} \psi+\frac{1}{2 \sqrt{2}} T \bar{\psi} \psi\right] .
$$

The action (2.10) is obtained by the dimensional reduction and truncations of some fields from this one. In [19] by expanding the action around the kink solution

$$
T(x)=\sqrt{2} x^{9} \quad, \quad A_{\mu}(x)=0 \quad, \quad \psi(x)=0,
$$

which is similar to (2.7), the authors investigate the spectrum on this soliton. Note that the $T \bar{\psi} \psi$ term is like a mass term of domain wall type to produce chiral fermion. As a result the massless spectrum is the vector supermultiplet in 9 dimension, which is same as the one on the BPS D8-brane. The massive spectrums are a little different, while there are no continuous spectrums and the mass square differences are equal spacing, which is the similar structure as the one in string theory. However, in [19] the supersymmetry is not considered, thus we can not understand how the linear supersymmetry restores at a stable vacuum.

In this paper as a first step to understand the full structure of the nonlinear supersymmetry on a non-BPS D-brane, we will consider the several leading terms of the effective action and will confirm that the nonlinear supersymmetry is realized and that the action is consistent with the result from boundary string field theory.

\section{Construction of the Action with Nonlinear Super- symmetry}

In this section we will analyze the constraints which come from the supersymmetric invariance of the action and will show that the nonlinear supersymmetry can be realized on the effective action of a non-BPS D9-brane.

\footnotetext{
${ }^{7}$ It is not essential to consider the Euclidean action. Indeed, we will construct an analog of this model in four dimension in appendix A.
} 
First we consider the most general action and the supersymmetry transformation which are constructed by $T(x), A_{\mu}(x)$ and $\psi(x)$ up to several order. Here we pick up the terms which satisfy the following conditions:

$$
\# A_{\mu}(x)=0, \quad \# \psi(x)=1 \text { and } \# \partial_{\mu} \leq 1 \text { in } \delta S
$$

where $\# X$ is the number of $X$ and $\delta S$ is the variation of the action by the supersymmetry transformation. Of course, the following analysis can be done by using the $\alpha^{\prime}$ expansion in the same way. Under this condition the relevant terms are as follows

$$
\begin{aligned}
S= & -\sqrt{2} T_{9} \int d^{10} x e^{-\frac{1}{4} T^{2}} \\
& \times\left[1+f_{1}(T)(\bar{\psi} \psi)+f_{2}(T)\left(\bar{\psi} \Gamma^{\mu} \partial_{\mu} \psi\right)+f_{3}(T)\left(\bar{\psi} \Gamma_{11} \Gamma^{\mu} \partial_{\mu} \psi\right)\right] .
\end{aligned}
$$

And we also consider the supersymmetry transformations

$$
\begin{aligned}
\delta \psi_{\alpha}= & g_{1}(T) \epsilon_{\alpha}+g_{2}(T)\left(\Gamma_{11} \epsilon\right)_{\alpha}+g_{3}(T) \partial^{\mu} T\left(\Gamma_{\mu} \epsilon\right)_{\alpha}+g_{4}(T) \partial^{\mu} T\left(\Gamma_{11} \Gamma_{\mu} \epsilon\right)_{\alpha}, \\
\delta T= & h_{1}(T)(\bar{\epsilon} \psi)+h_{2}(T)\left(\bar{\epsilon} \Gamma_{11} \psi\right)+h_{3}(T) \partial^{\mu} T\left(\bar{\epsilon} \Gamma_{\mu} \psi\right) \\
& +h_{4}(T) \partial^{\mu} T\left(\bar{\epsilon} \Gamma_{11} \Gamma_{\mu} \psi\right)+h_{5}(T)\left(\bar{\epsilon} \Gamma^{\mu} \partial_{\mu} \psi\right)+h_{6}(T)\left(\bar{\epsilon} \Gamma_{11} \Gamma^{\mu} \partial_{\mu} \psi\right),
\end{aligned}
$$

where the coefficient functions $f_{i}(T), g_{i}(T)$ and $h_{i}(T)$ are nonsingular around $T=0$. Now we will consider the constraints which come from the supersymmetric invariance of the action (3.17), that is, $\delta S=0$. These are the relations between the coefficient functions:

$$
\begin{aligned}
\delta S= & -\sqrt{2} T_{9} \int d^{10} x e^{-\frac{1}{4} T^{2}} \\
& \times\left[C_{1}(T)(\bar{\epsilon} \psi)+C_{2}(T)\left(\bar{\epsilon} \Gamma_{11} \psi\right)+C_{3}(T) \partial^{\mu} T\left(\bar{\epsilon} \Gamma_{\mu} \psi\right)+C_{4}(T) \partial^{\mu} T\left(\bar{\epsilon} \Gamma_{11} \Gamma_{\mu} \psi\right)\right]
\end{aligned}
$$

where

$$
\begin{aligned}
C_{1}= & -\frac{1}{2} T h_{1}+2 f_{1} g_{1} \quad, \quad C_{2}=-\frac{1}{2} T h_{2}-2 f_{1} g_{2}, \\
C_{3}= & \frac{1}{2} T f_{2} g_{1}-2 f_{1} g_{3}-\frac{1}{2} T h_{3}-2 f_{2} \dot{g}_{1}+2 f_{3} \dot{g}_{2} \\
& +\left(\frac{1}{2}-\frac{1}{4} T^{2}\right) h_{5}-\frac{1}{2} T f_{3} g_{2}+\frac{1}{2} T \dot{h}_{5}-\dot{f}_{2} g_{1}+\dot{f}_{3} g_{2}, \\
C_{4}= & -\frac{1}{2} T f_{2} g_{2}-2 f_{1} g_{4}-\frac{1}{2} T h_{4}+2 f_{2} \dot{g}_{2}-2 f_{3} \dot{g}_{1} \\
& +\left(\frac{1}{2}-\frac{1}{4} T^{2}\right) h_{6}+\frac{1}{2} T f_{3} g_{1}+\frac{1}{2} T \dot{h}_{6}+\dot{f}_{2} g_{2}-\dot{f}_{3} g_{1},
\end{aligned}
$$

and $\dot{f}$ denotes $d f / d T$. From this we can obtain four constraint equations $C_{i}(T)=0(i=$ $1,2,3,4)$.

However, only with these conditions we can not completely fix the functions $f_{i}(T), g_{i}(T)$ and $h_{i}(T)$. In general the effective action and the transformations of fields which represent 
some symmetries have the field redefinition ambiguities of the realization. Of course the field redefinition does not change the physics. Especially in the boundary string field theory this ambiguity comes from the regularization of the nonlinear sigma model. Therefore also in this case we have to consider the most general field redefinition of $T(x)$ and $\psi(x)$ which are nonsingular around $T=0$, and fix these ambiguities.

The field redefinitions which are needed in (3.17), (3.18) and (3.19) are

$$
\begin{aligned}
\psi^{\prime}= & k_{1}(T) \psi_{\alpha}+k_{2}(T)\left(\Gamma_{11} \psi\right)_{\alpha}+k_{3}(T) \partial^{\mu} T\left(\Gamma_{\mu} \psi\right)_{\alpha}+k_{4}(T) \partial^{\mu} T\left(\Gamma_{11} \Gamma_{\mu} \psi\right)_{\alpha} \\
& +k_{5}(T)\left(\Gamma^{\mu} \partial_{\mu} \psi\right)_{\alpha}+k_{6}(T)\left(\Gamma_{11} \Gamma^{\mu} \partial_{\mu} \psi\right)_{\alpha}, \\
T^{\prime}= & T+l_{1}(T)(\bar{\psi} \psi)+l_{2}(T)\left(\bar{\psi} \Gamma^{\mu} \partial_{\mu} \psi\right)+l_{3}(T)\left(\bar{\psi} \Gamma_{11} \Gamma^{\mu} \partial_{\mu} \psi\right) .
\end{aligned}
$$

Moreover the parameter $\epsilon_{\alpha}$ of the supersymmetry transformation in (3.18) and (3.19) has the following ambiguity:

$$
\epsilon_{\alpha} \rightarrow a \epsilon_{\alpha}+b\left(\Gamma_{11} \epsilon\right)_{\alpha}
$$

where $a, b$ are constants. We have fixed the first term of eq(3.22) to $T$, not general function of $T$. This is because $T \rightarrow f(T)$ changes the potential. We have fixed the potential to $e^{-\frac{1}{4} T^{2}}$ which was obtained by boundary string field theory [6, 7].

From (3.17), (3.18) and (3.19) we can see that there are 13 coefficient functions $f_{i}(T), g_{i}(T)$ and $h_{i}(T)$ which are not determined. On the other hand (3.20) gives 4 constraint equations which come from the supersymmetry, and (3.21) and (3.22) represent the 9 freedoms of the field redefinitions. Therefore we expect that all coefficient functions $f_{i}(T), g_{i}(T)$ and $h_{i}(T)$ can be determined completely.

In fact we can do. At first order the coefficients $g_{1}(T)$ and $g_{2}(T)$ in eq(3.18) transforms as follows:

$$
g_{1} \rightarrow k_{1} g_{1}+k_{2} g_{2} \quad, \quad g_{2} \rightarrow k_{1} g_{2}+k_{2} g_{1}
$$

From these we can consistently fix $g_{1}(T)$ and $g_{2}(T)$ to 1 and 0 , respectively. By representing the action and the supersymmetry transformations with the redefined fields $\psi^{\prime}(x)$ and $T^{\prime}(x)$, the coefficient functions $f_{i}(T), g_{i}(T)$ and $h_{i}(T)$ transform as follows: ${ }^{8}$

$$
\begin{aligned}
& f_{1} \rightarrow f_{1}+\frac{1}{2} T l_{1}, \\
& f_{2} \rightarrow f_{2}-2 f_{1} k_{5}+\frac{1}{2} T l_{2}-T l_{1} k_{5} \quad, \quad f_{3} \rightarrow f_{3}-2 f_{1} k_{6}+\frac{1}{2} T l_{3}-T l_{1} k_{6}, \\
& g_{3} \rightarrow g_{3}+k_{3} \quad, \quad g_{4} \rightarrow g_{4}+k_{4} \quad, \quad h_{1} \rightarrow h_{1}+2 l_{1} \quad, \quad h_{2} \rightarrow h_{2},
\end{aligned}
$$

${ }^{8}$ Sting theory should keep G-parity invariance before and after interactions. From this we can restrict all the coefficient functions $f_{i}(T), g_{i}(T), h_{i}(T), k_{i}(T)$ and $l_{i}(T)$ to the even or odd functions of $\mathrm{T}$. 


$$
\begin{aligned}
& h_{3} \rightarrow h_{3}-2 l_{1} g_{3}-k_{3} h_{1}-2 k_{3} l_{1}-k_{4} h_{2}, \\
& h_{4} \rightarrow h_{4}-2 l_{1} g_{4}-k_{4} h_{1}-2 k_{4} l_{1}-k_{3} h_{2}, \\
& h_{5} \rightarrow h_{5}-k_{5} h_{1}-2 k_{5} l_{1}-k_{6} h_{2} \quad, \quad h_{6} \rightarrow h_{6}-k_{6} h_{1}-2 k_{6} l_{1}-k_{5} h_{2} .
\end{aligned}
$$

Therefore from these equations we can consistently fix the coefficient functions and obtain the following results: ${ }^{9}$

$$
\begin{gathered}
S=-\sqrt{2} T_{9} \int d^{10} x e^{-\frac{1}{4} T^{2}}\left[1+\frac{1}{2 \sqrt{2}} T(\bar{\psi} \psi)-\left(\bar{\psi} \Gamma^{\mu} \partial_{\mu} \psi\right)\right], \\
\delta \psi_{\alpha}=\epsilon_{\alpha}+\frac{1}{\sqrt{2}} \partial^{\mu} T\left(\Gamma_{\mu} \epsilon\right)_{\alpha} \quad, \quad \delta T=\sqrt{2}(\bar{\epsilon} \psi)-2 \partial^{\mu} T\left(\bar{\epsilon} \Gamma_{\mu} \psi\right) .
\end{gathered}
$$

From this result we find that we can obtain the same action as the one considered in [19 by appropriate fixing of the field redefinition ambiguities. Also from $\delta \psi(x)=0$ we can obtain the same $1 / 2$-BPS solutions as (2.15). For this solution the $1 / 2$ supersymmetry which satisfy $\Gamma^{9} \epsilon=-\epsilon$ restores on the BPS D-brane. If we again redefine the new field in order to normalize the kinetic term to 1, then the exponential potential appears in front of the right-hand side of (3.26) and we obtain another BPS solution $T \rightarrow \pm \infty$ which represents the closed string vacuum in the same way as (2.13). The above result indicates that the structure of the supersymmetry can be included in the action of [19].

From this explicit practice we obtained the effective action with the nonlinear supersymmetry at the leading order. However in this order the gauge fields are not introduced. Therefore we will extend this result to the effective action with more terms which satisfy the following conditions.

$$
\# A_{\mu}(x) \leq 1, \quad \# \psi(x)=1 \text { and } \# \partial_{\mu} \leq 2 \text { in } \delta S
$$

where $\# X$ is the number of $X$. In the appendix $\mathrm{C}$ we write the general action, the supersymmetry transformations and the field redefinitions up to the above order. The process to determine the coefficient functions of the action and the supersymmetry transformations is same as the previous one, therefore we abbreviate this process. We fix the field redefinition ambiguities appropriately. The final form of the action is एण्ग

$$
S=-\sqrt{2} T_{9} \int d^{10} x e^{-\frac{1}{4} T^{2}}
$$

\footnotetext{
${ }^{9}$ Strictly speaking if we fix $h_{1}(T)$ to zero, we can not fix $h_{3}(T), h_{4}(T), h_{5}(T)$ and $h_{6}(T)$.

${ }^{10}$ The fact is that the total number of the constraints from the supersymmetric invariance and the field redefinition ambiguities (C.15) and (C.16) is a little more than the freedoms which appear in the action (C.11) and the supersymmetry transformations (C.12), (C.13) and (C.14). Nevertheless we find that various coefficient functions can be fixed with keeping the supersymmetry.

${ }^{11}$ We can change the coefficient of the term $\partial^{\mu} T \partial_{\mu} T$ in the action by fixing the field redefinition ambiguities differently. Therefore it is also possible to take another fixing to produce the boundary string field theory action (2.5).
} 


$$
\begin{aligned}
& \times\left[1+\frac{1}{2}\left(\partial^{\mu} T \partial_{\mu} T\right)+\frac{1}{2 \sqrt{2}} T(\bar{\psi} \psi)+\frac{1}{4} F^{\mu \nu} F_{\mu \nu}-\left(\bar{\psi} \Gamma^{\mu} \partial_{\mu} \psi\right)\right. \\
& \left.-2 \sqrt{2} \partial^{\mu} T\left(\bar{\psi} \partial_{\mu} \psi\right)+F^{\mu \nu}\left(\bar{\psi} \Gamma_{11} \Gamma_{\mu} \partial_{\nu} \psi\right)\right]
\end{aligned}
$$

and the supersymmetry transformations are

$$
\begin{aligned}
\delta \psi & =\epsilon+\frac{1}{\sqrt{2}} \partial^{\mu} T\left(\Gamma_{\mu} \epsilon\right)_{\alpha}, \\
\delta T & =\sqrt{2}(\bar{\epsilon} \psi)-2 \partial^{\mu} T\left(\bar{\epsilon} \Gamma_{\mu} \psi\right)-\sqrt{2} \partial^{\mu} T \partial_{\mu} T(\bar{\epsilon} \psi), \\
\delta A_{\mu} & =\left(\bar{\epsilon} \Gamma_{11} \Gamma_{\mu} \psi\right)
\end{aligned}
$$

Thus we find that there exists the nonlinear supersymmetry up to this order.

Then we have to verify whether these transformations of $\psi(x), T(x)$ and $A_{\mu}(x)$ satisfy the supersymmetry algebra. The expected forms of the supersymmetry algebra are

$$
\begin{aligned}
{\left[\delta_{1}, \delta_{2}\right] \psi } & =2\left(\bar{\epsilon}_{1} \Gamma^{\mu} \epsilon_{2}\right) \partial_{\mu} \psi \\
{\left[\delta_{1}, \delta_{2}\right] T } & =2\left(\bar{\epsilon}_{1} \Gamma^{\mu} \epsilon_{2}\right) \partial_{\mu} T \\
{\left[\delta_{1}, \delta_{2}\right] A_{\mu} } & =-2\left(\bar{\epsilon}_{1} \Gamma_{11} \Gamma_{\mu} \epsilon_{2}\right)+2\left(\bar{\epsilon}_{1} \Gamma^{\rho} \epsilon_{2}\right) \partial_{\rho} A_{\mu}
\end{aligned}
$$

We consider that for $\psi(x)$ and $A_{\mu}(x)$ the algebras will be same as (2.4). Indeed if we repeat supersymmetry transformations (3.29) again, we can obtain the same result up to the reliable order under the condition (3.27). For example, the translation term $2\left(\bar{\epsilon}_{1} \Gamma^{\mu} \epsilon_{2}\right) \partial_{\mu} \psi$ in $\left[\delta_{1}, \delta_{2}\right] \psi$ does not appear from (3.29). However, by including the more higher terms about fermions in the action (3.28) and the supersymmetry transformations (3.29) we expect that this term appears correctly. The point of this discussion is that the form of the supersymmetry transformation is changed by the field redefinitions, while the supersymmetry algebra is invariant. $\mathrm{T}$. On the other hand, if we expand $T(x)$ around the solution (2.7) or (2.15) such as

$$
T(x)=u\left(x^{9}-X^{9}\left(x^{i}\right)\right)(u=\infty \text { or } \sqrt{2} ; i=0, \cdots, 8),
$$

then the algebra of the $X^{9}$ becomes the following one:

$$
\left[\delta_{1}, \delta_{2}\right] X^{9}=-2\left(\bar{\epsilon}_{1} \Gamma^{9} \epsilon_{2}\right)+2\left(\bar{\epsilon}_{1} \Gamma^{i} \epsilon_{2}\right) \partial_{i} X^{9}
$$

This algebra coincides with that of the transverse scalar on a BPS D8-brane [15, 10]. This is a desired result because $X^{9}\left(x^{i}\right)$ is the translation mode of the soliton (BPS D8 brane) [20, 19] as we can see from (3.31).

\footnotetext{
${ }^{12}$ There are terms from the gauge transformation and terms proportional to the equations of motion in (3.30).
} 


\section{Conclusions and Discussions}

In this paper we introduced the boundary string field theory action and constructed the action order-by-order which includes fermions and realizes the nonlinear supersymmetry. In the first practice we obtained the same action as the Minahan-Zwiebach model [19] and verified that the nonlinear supersymmetry can be realized. Next in order to include the gauge field, we extend this action to the one with more higher dimensional terms. Then we found that we can construct the action with the nonlinear supersymmetry which is consistent with the boundary string field theory action, and confirmed that this action can have the BPS soliton solution in the supersymmetric sense, which is similar to the one (2.7) in the boundary string field theory.

However, any forms of the action are allowed in the above order, therefore the definite conclusions are not obtained as far as we consider the finite numbers of terms in the action and supersymmetry transformations. As an example let us consider the BPS solution. From the existence of the solution (2.7) in boundary string field theory, we expect that the form of $\delta \psi(x)$ is the following:

$$
\begin{aligned}
\delta \psi_{\alpha}= & L\left(T^{2}\right)\left[M\left(\partial^{\mu} T \partial_{\mu} T\right) \epsilon_{\alpha}+N\left(\partial^{\mu} T \partial_{\mu} T\right) \partial^{\nu} T\left(\Gamma_{\nu} \epsilon\right)_{\alpha}\right] \\
& +\left(\text { terms including } \psi, A_{\mu}, \partial_{\mu} \partial_{\nu} T\right)
\end{aligned}
$$

where $L(x), M(x)$ and $N(x)$ are nonsingular functions around $x=0$, with a relation

$$
\lim _{x \rightarrow \infty}\left|x N\left(x^{2}\right) / M\left(x^{2}\right)\right|=1 .
$$

Note that here the fields are partially fixed according to the boundary string field theory, and in that sense (4.33) depends on the remaining field redefinitions. Then if we truncate the above transformation such as (3.18), we are able to set the coefficients $g_{i}(T)(i=2,3,4)$ to zero by the field redefinitions (see (3.24), (3.25)). From this form we can not see the BPS solution explicitly. The analysis of truncated actions (3.26) and (3.28) showed that at least the realization of the nonlinear supersymmetry does not contradict the existence of the BPS solutions.

One of the motivations of this paper was from the fact that the truncated action in [19 is a good approximation. However, because the mass of the tachyon is the order of the Planck scale, it is difficult to consider the reason why such a truncated action can work for the tachyon condensation. Any way, to find the full order action with the nonlinear supersymmetry we consider that some other algebraic principle to construct the action should be needed.

As a further study of the supersymmetry, it is interesting to extend the analysis in this paper to the non-Abelian non-BPS D-branes because also in this case the nontrivial and exact BPS solution is already known [21]. There are a lot of things to investigate. 


\section{Acknowledgements}

We would like to thank T. Hara, K. Ohmori and T. Takayanagi for useful discussions. The works of S.T. were supported in part by JSPS Research Fellowships for Young Scientists.

\section{A A Four Dimensional Model with Nonlinear Super- symmetry}

Here we introduce a four dimensional toy model with the nonlinear supersymmetry using the four dimensional $N=1$ superfield formulation. We will use the notation used in [22]. We consider a chiral superfield $\Phi(x, \theta)=T(x)+i a(x)+\sqrt{2} \theta \chi+\cdots$ with a Lagrangian

$$
L=\int d^{2} \theta d^{2} \bar{\theta} K(\Phi, \bar{\Phi})+\left[\int d^{2} \theta P(\Phi)+\text { h.c. }\right]
$$

where the Kähler potential $K$ and the superpotential $P$ are given by

$$
K(\Phi, \bar{\Phi})=\left|\int_{0}^{\Phi} d y e^{-\frac{1}{8} y^{2}}\right|^{2}, P(\Phi)=i \int_{0}^{\Phi} d y e^{-\frac{1}{4} y^{2}} .
$$

Then the metric on the Kähler manifold becomes

$$
g_{\Phi \bar{\Phi}}=e^{-\frac{1}{8}\left(\Phi^{2}+\bar{\Phi}^{2}\right)}
$$

$\Gamma_{\Phi \Phi}^{\Phi}=-\frac{1}{4} \Phi$ and $R_{\Phi \bar{\Phi} \Phi \bar{\Phi}}=0$. Then the component Lagrangian is computed as

$$
\begin{gathered}
L=-e^{-\frac{T^{2}}{4}} e^{\frac{a^{2}}{4}}\left\{1+\partial_{m} T \partial^{m} T+\partial_{m} a \partial^{m} a+i \bar{\chi} \bar{\sigma}^{m} \partial_{m} \chi-i \bar{\chi} \bar{\sigma}^{m} \chi \frac{1}{4}(T+i a) \partial_{m}(T+i a)\right. \\
\left.+i \frac{1}{8}(T+i a) e^{-i \frac{1}{2} a T} \chi \chi-i \frac{1}{8}(T-i a) e^{+i \frac{1}{2} a T} \bar{\chi} \bar{\chi}\right\}
\end{gathered}
$$

which has a nonlinearly realized supersymmetry at $T=a=0$ given by

$$
\delta(T+i a)=\sqrt{2} \xi \chi, \quad \delta \chi=i \sqrt{2} \sigma^{m} \bar{\xi} \partial_{m}(T+i a)+\frac{1}{4}(T+i a) \delta(T+i a) \chi-i \sqrt{2} e^{i \frac{1}{2} a T} \xi .
$$

This model has a $1 / 2$-BPS domain wall solution $T=x^{3}$ and $a=\xi=0$.

If we identify the real scalar $T$ as the tachyon, this action can be regarded as a toy model for the tachyon in a non-BPS D-brane. Indeed, the model has the characteristic properties such as the restoration of the supersymmetry at $T= \pm \infty$ where the action vanishes and the existence of a $1 / 2$-BPS solution corresponding to a one lower dimensional BPS D-brane.

This model can be extended to other dimensions and to other matter contents. 


\section{B $\quad \mathrm{SO}(1,9)$ Clifford Algebra}

The gamma matrices $\Gamma^{\mu}(\mu=0,1,2, \cdots, 9)$ are defined by

$$
\left\{\Gamma^{\mu}, \Gamma^{\nu}\right\}=2 \eta^{\mu \nu} .
$$

Here the sign convention of the flat metric $\eta^{\mu \nu}$ is most plus one, that is, $\operatorname{diag}\left(\eta^{\mu \nu}\right)=$ $(-,+,+\cdots,+)$. We define $\Gamma_{11}$ as

$$
\Gamma_{11} \equiv \Gamma^{0} \Gamma^{1} \cdots \Gamma^{9},
$$

and the charge conjugation matrix $C$ as

$$
\begin{aligned}
\bar{\psi}_{\alpha} \equiv-\psi_{\beta}(C)_{\beta \alpha}, \\
C^{T}=-C, \quad C \Gamma^{\mu} C^{-1}=-\left(\Gamma^{\mu}\right)^{T}, \\
C \Gamma_{11} C^{-1}=-\left(\Gamma_{11}\right)^{T} .
\end{aligned}
$$

Then the symmetry of matrices is the following:

$$
\begin{array}{rll}
\text { Symmetric matrices } & \cdots & C \Gamma^{\mu}, C \Gamma^{\mu \nu}, C \Gamma^{\mu_{1} \cdots \mu_{5}}, C \Gamma_{11}, C \Gamma_{11} \Gamma^{\mu}, C \Gamma_{11} \Gamma^{\mu_{1} \cdots \mu_{4}}, \\
\text { Antisymmetric matrices } & \cdots & C, C \Gamma^{\mu_{1} \mu_{2} \mu_{3}}, C \Gamma^{\mu_{1} \cdots \mu_{4}}, C \Gamma_{11} \Gamma^{\mu \nu}, C \Gamma_{11} \Gamma^{\mu_{1} \mu_{2} \mu_{3}} .
\end{array}
$$

From this we can derive the following identities:

$$
\begin{array}{r}
\bar{\psi} \Gamma^{\mu} \psi=\bar{\psi} \Gamma^{\mu \nu} \psi=\bar{\psi} \Gamma^{\mu_{1} \mu_{2} \cdots \mu_{5}} \psi=0 \\
\bar{\psi} \Gamma^{11} \psi=\bar{\psi} \Gamma^{11} \Gamma^{\mu} \psi=\bar{\psi} \Gamma^{11} \Gamma^{\mu_{1} \mu_{2} \cdots \mu_{4}} \psi=0
\end{array}
$$

\section{General Effective Action, Supersymmetry Trans- formations and Field Redefinitions}

Here we write all relevant terms which obey (3.27) in the action, the supersymmetry transformations and the field redefinitions of $\psi(x), T(x)$ and $A_{\mu}(x)$. The coefficients $f_{i}, g_{i}, h_{i}, j_{i}, k_{i}$ and $l_{i}$ which appear in the latter equations are the nonsingular functions of $T$ around $T=0$, while $m_{1}$ is a constant.

The action:

$$
\begin{aligned}
S= & -\sqrt{2} T_{9} \int d^{10} x e^{-\frac{1}{4} T^{2}} \\
\times & {\left[1+f_{1}(\bar{\psi} \psi)+f_{2}\left(\bar{\psi} \Gamma^{\mu} \partial_{\mu} \psi\right)+f_{3}\left(\bar{\psi} \Gamma_{11} \Gamma^{\mu} \partial_{\mu} \psi\right)+f_{4}\left(\partial^{\mu} T \partial_{\mu} T\right)+f_{5} F^{\mu \nu} F_{\mu \nu}\right.} \\
& +f_{6} F^{\mu \nu}\left(\bar{\psi} \Gamma_{11} \Gamma_{\mu \nu} \psi\right)+f_{7}\left(\overline{\partial^{\mu} \psi} \partial_{\mu} \psi\right)+f_{8} \partial^{\mu} T\left(\bar{\psi} \partial_{\mu} \psi\right)+f_{9}\left(\partial^{\mu} T \partial_{\mu} T\right)(\bar{\psi} \psi) \\
& +f_{10} \partial^{\mu} T\left(\bar{\psi} \Gamma_{11} \partial_{\mu} \psi\right)+f_{11} F^{\mu \nu}\left(\bar{\psi} \Gamma_{\mu} \partial_{\nu} \psi\right)+f_{12} F^{\mu \nu}\left(\bar{\psi} \Gamma_{11} \Gamma_{\mu} \partial_{\nu} \psi\right) \\
& \left.+f_{13} F^{\mu \nu}\left(\bar{\psi} \Gamma_{\mu \nu \rho} \partial^{\rho} \psi\right)+f_{14} F^{\mu \nu}\left(\bar{\psi} \Gamma_{11} \Gamma_{\mu \nu \rho} \partial^{\rho} \psi\right)\right] .
\end{aligned}
$$


The supersymmetry transformations:

$$
\begin{aligned}
\delta \psi_{\alpha}= & g_{1} \epsilon_{\alpha}+g_{2}\left(\Gamma_{11} \epsilon\right)_{\alpha}+g_{3} \partial^{\mu} T\left(\Gamma_{\mu} \epsilon\right)_{\alpha}+g_{4} \partial^{\mu} T\left(\Gamma_{11} \Gamma_{\mu} \epsilon\right)_{\alpha} \\
& +g_{5} F^{\mu \nu}\left(\Gamma_{\mu \nu} \epsilon\right)_{\alpha}+g_{6} F^{\mu \nu}\left(\Gamma_{11} \Gamma_{\mu \nu} \epsilon\right)_{\alpha}+g_{7}\left(\partial^{\mu} T \partial_{\mu} T\right) \epsilon_{\alpha} \\
& +g_{8}\left(\partial^{\mu} T \partial_{\mu} T\right)\left(\Gamma_{11} \epsilon\right)_{\alpha}+g_{9} \partial^{2} T \epsilon_{\alpha}+g_{10} \partial^{2} T\left(\Gamma_{11} \epsilon\right)_{\alpha} \\
& +g_{11} F^{\mu \nu} \partial_{\mu} T\left(\Gamma_{\nu} \epsilon\right)_{\alpha}+g_{12} F^{\mu \nu} \partial_{\mu} T\left(\Gamma_{11} \Gamma_{\nu} \epsilon\right)+g_{13} \partial_{\mu} F^{\mu \nu}\left(\Gamma_{\nu} \epsilon\right)_{\alpha} \\
& +g_{14} \partial_{\mu} F^{\mu \nu}\left(\Gamma_{11} \Gamma_{\nu} \epsilon\right)_{\alpha}+g_{15} F^{\mu \nu} \partial^{\rho} T\left(\Gamma_{\mu \nu \rho} \epsilon\right)+g_{16} F^{\mu \nu} \partial^{\rho} T\left(\Gamma_{11} \Gamma_{\mu \nu \rho} \epsilon\right)_{\alpha},( \\
\delta T= & h_{1}(\bar{\epsilon} \psi)+h_{2}\left(\bar{\epsilon} \Gamma_{11} \psi\right)+h_{3} \partial^{\mu} T\left(\bar{\epsilon} \Gamma_{\mu} \psi\right)+h_{4} \partial^{\mu} T\left(\bar{\epsilon} \Gamma_{11} \Gamma_{\mu} \psi\right) \\
+ & h_{5}\left(\bar{\epsilon} \Gamma^{\mu} \partial_{\mu} \psi\right)+h_{6}\left(\bar{\epsilon} \Gamma_{11} \Gamma^{\mu} \partial_{\mu} \psi\right)+h_{7} F^{\mu \nu}\left(\bar{\epsilon} \Gamma_{\mu \nu} \psi\right) \\
+ & h_{8} F^{\mu \nu}\left(\bar{\epsilon} \Gamma_{11} \Gamma_{\mu \nu} \psi\right)+h_{9} \partial^{\mu} T\left(\bar{\epsilon} \partial_{\mu} \psi\right)+h_{10}\left(\bar{\epsilon} \partial^{2} \psi\right) \\
+ & h_{11} \partial^{2} T(\bar{\epsilon} \psi)+h_{12} \partial^{\mu} T \partial_{\mu} T(\bar{\epsilon} \psi)+h_{13} \partial^{\mu} T\left(\bar{\epsilon} \Gamma_{11} \partial_{\mu} \psi\right)+h_{14}\left(\bar{\epsilon} \Gamma_{11} \partial^{2} \psi\right) \\
+ & h_{15} \partial^{2} T\left(\bar{\epsilon} \Gamma_{11} \psi\right)+h_{16} \partial^{\mu} T \partial_{\mu} T\left(\bar{\epsilon} \Gamma_{11} \psi\right)+h_{17} F^{\mu \nu} \partial_{\mu} T\left(\bar{\epsilon} \Gamma_{\nu} \psi\right) \\
+ & h_{18} F^{\mu \nu} \partial_{\mu} T\left(\bar{\epsilon} \Gamma_{11} \Gamma_{\nu} \psi\right)+h_{19} \partial_{\mu} F^{\mu \nu}\left(\bar{\epsilon} \Gamma_{\nu} \psi\right)+h_{20} \partial_{\mu} F^{\mu \nu}\left(\bar{\epsilon} \Gamma_{11} \Gamma_{\nu} \psi\right) \\
+ & h_{21} F^{\mu \nu}\left(\bar{\epsilon} \Gamma_{\mu} \partial_{\nu} \psi\right)+h_{22} F^{\mu \nu}\left(\bar{\epsilon} \Gamma_{11} \Gamma_{\mu} \partial_{\nu} \psi\right)+h_{23} F^{\mu \nu} \partial^{\rho} T\left(\bar{\epsilon} \Gamma_{\mu \nu \rho} \psi\right) \\
+ & h_{24} F^{\mu \nu} \partial^{\rho} T\left(\bar{\epsilon} \Gamma_{11} \Gamma_{\mu \nu \rho} \psi\right)+h_{25} F^{\mu \nu}\left(\bar{\epsilon} \Gamma_{\mu \nu \rho} \partial^{\rho} \psi\right)+h_{26} F^{\mu \nu}\left(\bar{\epsilon} \Gamma_{11} \Gamma_{\mu \nu \rho} \partial^{\rho} \psi\right),(\mathrm{C} . \\
\delta & j_{1}\left(\bar{\epsilon} \Gamma_{\mu} \psi\right)+j_{2}\left(\bar{\epsilon} \Gamma_{11} \Gamma_{\mu} \psi\right) .
\end{aligned}
$$

Field Redefinitions:

$$
\begin{aligned}
\psi^{\prime}= & k_{1} \psi_{\alpha}+k_{2}\left(\Gamma_{11} \psi\right)_{\alpha}+k_{3} \partial^{\mu} T\left(\Gamma_{\mu} \psi\right)_{\alpha}+k_{4} \partial^{\mu} T\left(\Gamma_{11} \Gamma_{\mu} \psi\right)_{\alpha} \\
& +k_{5}\left(\Gamma^{\mu} \partial_{\mu} \psi\right)_{\alpha}+k_{6}\left(\Gamma_{11} \Gamma^{\mu} \partial_{\mu} \psi\right)_{\alpha}+k_{7} F^{\mu \nu}\left(\Gamma_{\mu \nu} \psi\right)_{\alpha}+k_{8} F^{\mu \nu}\left(\Gamma_{11} \Gamma_{\mu \nu} \psi\right)_{\alpha} \\
& +k_{9}\left(\partial^{2} \psi\right)_{\alpha}+k_{10}\left(\Gamma_{11} \partial^{2} \psi\right)_{\alpha}+k_{11} \partial^{\mu} T\left(\partial_{\mu} \psi\right)_{\alpha}+k_{12} \partial^{\mu} T\left(\Gamma_{11} \partial_{\mu} \psi\right)_{\alpha} \\
& +k_{13} \partial^{2} T \psi_{\alpha}+k_{14} \partial^{2} T\left(\Gamma_{11} \psi\right)_{\alpha}+k_{15} \partial^{\mu} T \partial_{\mu} T \psi_{\alpha} \\
& +k_{16} \partial^{\mu} T \partial_{\mu} T\left(\Gamma_{11} \psi\right)_{\alpha}+k_{17} F^{\mu \nu} \partial_{\mu} T\left(\Gamma_{\nu} \psi\right)_{\alpha}+k_{18} F^{\mu \nu} \partial_{\mu} T\left(\Gamma_{11} \Gamma_{\nu} \psi\right)_{\alpha} \\
& +k_{19} \partial_{\mu} F^{\mu \nu}\left(\Gamma_{\nu} \psi\right)_{\alpha}+k_{20} \partial_{\mu} F^{\mu \nu}\left(\Gamma_{11} \Gamma_{\nu} \psi\right)_{\alpha}+k_{21} F^{\mu \nu}\left(\Gamma_{\mu} \partial_{\nu} \psi\right)_{\alpha} \\
& +k_{22} F^{\mu \nu}\left(\Gamma_{11} \Gamma_{\mu} \partial_{\nu} \psi\right)_{\alpha}+k_{23} F^{\mu \nu} \partial^{\rho} T\left(\Gamma_{\mu \nu \rho} \psi\right)_{\alpha}+k_{24} F^{\mu \nu} \partial^{\rho} T\left(\Gamma_{11} \Gamma_{\mu \nu \rho} \psi\right)_{\alpha} \\
& +k_{25} F^{\mu \nu}\left(\Gamma_{\mu \nu \rho} \partial^{\rho} \psi\right)_{\alpha}+k_{26} F^{\mu \nu}\left(\Gamma_{11} \Gamma_{\mu \nu \rho} \partial^{\rho} \psi\right)_{\alpha} \\
= & T+l_{1}(\bar{\psi} \psi)+l_{2}\left(\bar{\psi} \Gamma^{\mu} \partial_{\mu} \psi\right)+l_{3}\left(\bar{\psi} \Gamma_{11} \Gamma^{\mu} \partial_{\mu} \psi\right)+l_{4}\left(\partial^{\mu} T \partial_{\mu} T\right)+l_{5} \partial^{2} T \\
& +l_{6} F^{\mu \nu} F_{\mu \nu}+l_{7} F^{\mu \nu}\left(\bar{\psi} \Gamma_{11} \Gamma_{\mu \nu} \psi\right)+l_{8}\left(\bar{\psi} \partial^{2} \psi\right)+l_{9}\left(\overline{\partial^{\mu} \psi} \partial_{\mu} \psi\right) \\
& +l_{10} \partial^{\mu} T\left(\bar{\psi} \partial_{\mu} \psi\right)+l_{11} \partial^{2} T(\bar{\psi} \psi)+l_{12} \partial^{\mu} T \partial_{\mu} T(\bar{\psi} \psi) \\
& +l_{13}\left(\bar{\psi} \Gamma_{11} \partial^{2} \psi\right)+l_{14} \partial^{\mu} T\left(\bar{\psi} \Gamma_{11} \partial_{\mu} \psi\right)+l_{15} F^{\mu \nu}\left(\bar{\psi} \Gamma_{\mu} \partial_{\nu} \psi\right) \\
& +l_{16} F^{\mu \nu}\left(\bar{\psi} \Gamma_{11} \Gamma_{\mu} \partial_{\nu} \psi\right)+l_{17} F^{\mu \nu}\left(\bar{\psi} \Gamma_{\mu \nu \rho} \partial^{\rho} \psi\right)+l_{18} F^{\mu \nu}\left(\bar{\psi} \Gamma_{11} \Gamma_{\mu \nu \rho} \partial^{\rho} \psi\right) \\
& +l_{19} F^{\mu \nu} \partial^{\rho} T\left(\bar{\psi} \Gamma_{\mu \nu \rho} \psi\right)+l_{20} F^{\mu \nu} \partial^{\rho} T\left(\bar{\psi} \Gamma_{11} \Gamma_{\mu \nu \rho} \psi\right) \\
A_{\mu}^{\prime} & m_{1} A_{\mu} .
\end{aligned}
$$




\section{References}

[1] For a review, see A. Sen, "Non-BPS States and Branes in String Theory," hepth/9904207.

[2] M.R. Gaberdiel, "Lectures on Non-BPS Dirichlet Branes, "Class. Quant. Grav. 17 (2000) 3483, hep-th/0005029.

[3] E. Witten, "On background independent open string field theory," Phys. Rev. D46 (1992) 5467, hep-th/9208027; "Some computations in background independent offshell string theory," Phys. Rev. D47 (1993) 3405, hep-th/9210065.

[4] M. Marino, "On the BV formulation of boundary superstring field theory," hepth/0103089.

[5] V. Niarchos and N. Prezas, "Boundary Superstring Field Theory, "hep-th/0103102.

[6] D. Kutasov, M. Marino and G. Moore, "Remarks on tachyon condensation in superstring field theory," hep-th/0010108.

[7] A.A. Tseytlin, "Sigma model approach to string theory effective actions with tachyons," hep-th/0011033.

[8] P. Kraus and F. Larsen, "Boundary String Field Theory of the DDbar System," hep-th/0012198.

[9] T. Takayanagi, S. Terashima and T. Uesugi, "Brane-Antibrane Action from Boundary String Field Theory," JHEP 0003 (2001) 019, hep-th/0012210.

[10] A. Sen, "Supersymmetric World-volume Action for Non-BPS D-branes," JHEP 9910 (1999) 008, hep-th/9909062.

[11] T. Yoneya, "Spontaneously Broken Space-Time Supersymmetry in Open String Theory without GSO Projection," Nucl. Phys. B576 (2000) 219, hep-th/9912255.

[12] T. Hara and T. Yoneya, "Nonlinear Supersymmetry Without the GSO Projection and Unstable D9-Brane," hep-th/0010173.

[13] O. Andreev, "Some computations of partition functions and tachyon potentials in background independent off-shell string theory," Nucl. Phys. B 598 (2001) 151, hepth/0010218.

[14] K. Hashimoto and S. Hirano, "Metamorphosis Of Tachyon Profile In Unstable D9Branes," hep-th/0102174. 
[15] M. Aganagic, C. Popescu and J.H. Schwarz, "Gauge-Invariant and Gauge-Fixed DBrane Actions," Nucl. Phys. B495 (1997) 99, hep-th/9612080.

[16] G. Arutyunov, S. Frolov, S. Theisen and A.A. Tseytlin, "Tachyon condensation and universality of DBI action, " JHEP 0102 (2001) 002, hep-th/0012080.

[17] E. Witten, "Interacting Field Theory Of Open Superstrings," Nucl. Phys. B276 (1986) 291.

[18] F. Cooper, A. Khare and U. Sukhatme, "Supersymmetry and Quantum Mechanics," Physics Report 251 (1995) 267, hep-th/9405029.

[19] J.A. Minahan and B. Zwiebach, "Gauge Fields and Fermions in Tachyon Effective Field Theories, " JHEP 0102 (2001) 034, hep-th/0011226.

[20] J.A. Minahan and B. Zwiebach, "Effective tachyon dynamics in superstring theory," JHEP 0103 (2001) 038, hep-th/0009246.

[21] S. Terashima, "A Construction of Commutative D-branes from Lower Dimensional Non-BPS D-branes, "hep-th/0101087

[22] J. Wess and J. Bagger, "Supersymmetry and Supergravity," Princeton University Press. 